\title{
Hepatic tuberculosis: a rare cause of fluorodeoxyglucose hepatic superscan with background suppression on positron emission tomography
}

Simon Sin Man Wong${ }^{1}$, mBBS, Hok Yuen $\underline{\text { Yuen }}^{1}$, MBChB, FRCR, Anil T $\underline{\text { Ahuja }}^{1}$, MD, FRCR

\begin{abstract}
Fluorodeoxyglucose (FDG) hepatic superscan refers to the diffuse intense uptake of 18F-FDG in the liver on positron emission tomography (PET), with reduced physiological activity in the brain and heart. The common causes include lymphoma and metastasis. In this case report, we describe the imaging features of tuberculosis as a rare cause of FDG hepatic superscan. PET imaging may be the only clue to a diagnosis of hepatic tuberculosis, as other imaging modalities may demonstrate only nonspecific hepatomegaly. It is important to consider this entity in the differential diagnosis of patients presenting with FDG hepatic superscan and proceed with liver biopsy for a definitive diagnosis.
\end{abstract}

Keywords: 18F-FDG, liver, lymphoma, positron-emission tomography, tuberculosis

\section{INTRODUCTION}

Diffuse intense 18F-fluorodeoxyglucose (18F-FDG) uptake in the liver on positron emission tomography (PET), with reduced physiological activity in the brain and heart, is commonly known as FDG hepatic superscan. An uncommon finding on PET imaging, its causes are usually neoplastic, especially lymphoma. Herein we describe hepatic tuberculosis as a rare cause of FDG hepatic superscan.

\section{CASE REPORT}

A 46-year-old man with a history of hypertension, hyperlipidaemia and stroke presented with fever lasting four weeks. Physical examination showed mild hepatosplenomegaly. The chest was clear, and no skin rash or arthritis was evident. Laboratory tests revealed pancytopenia (haemoglobin, $7.6 \mathrm{~g} / \mathrm{dL}$; white blood cell count, $2.2 \times 10^{9} / \mathrm{L}$; platelet count, $139 \times 10^{9} / \mathrm{L}$ ), raised alkaline phosphatase (345 IU/L), and normal alanine aminotransferase and bilirubin levels.

Ultrasonography of the hepatobiliary system revealed diffuse increase in the liver parenchymal echotexture (Fig. 1). However, no focal hepatic lesion was identified. The spleen was also mildly enlarged, with a bipolar distance of $12.8 \mathrm{~cm}$ (Fig. 2). No evidence of biliary dilatation was noted. Magnetic resonance cholangiopancreaticography showed hepatosplenomegaly with no evidence of biliary obstruction. Blood, sputum and urine cultures were negative. Tests for hepatitis B surface antigen, anti-hepatitis $C$ virus antibody, anti-human immunodeficiency virus antibody and anti-Rickettsial antibody, Widal test and WeilFelix test were all negative. Bone marrow biopsy showed active marrow without abnormal cellular infiltrates. No septic focus was identified even after one week of hospitalisation.

In view of the pyrexia of unknown origin, PET imaging was performed, and it showed mild hepatosplenomegaly with diffuse

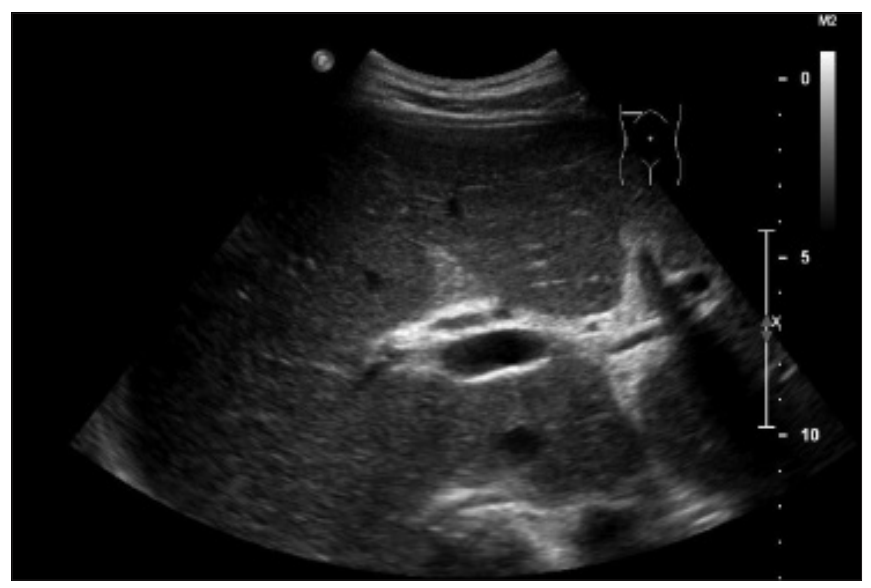

Fig. 1: US image of the hepatobiliary system shows mild hepatomegaly with diffuse increase in liver parenchymal echotexture. No focal hepatic lesion is identified.

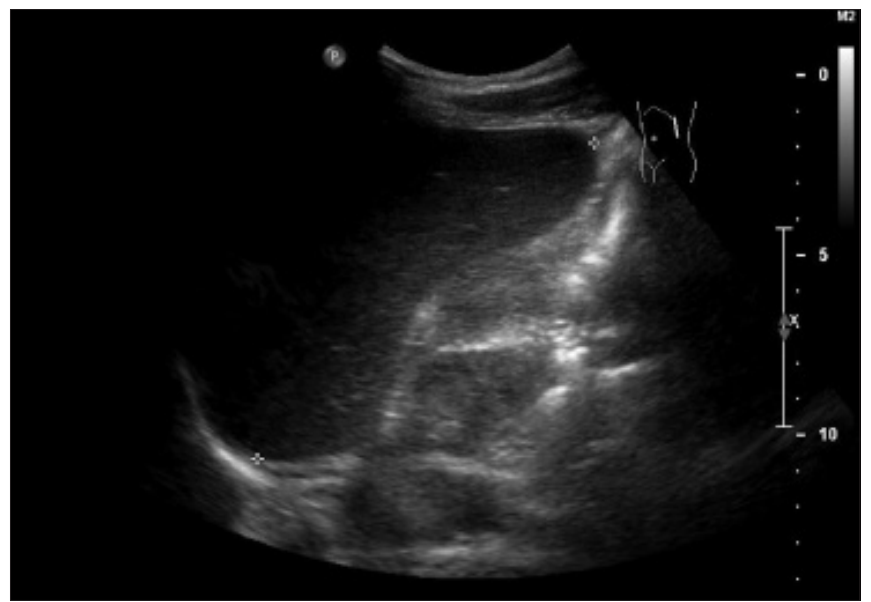

Fig. 2 US image of the spleen shows mild splenomegaly, with a bipolar distance of $12.8 \mathrm{~cm}$.

intense homogeneous FDG uptake in the liver (SUVmax, 6.6) (Fig. 3). No focal hepatic lesion was detected and no abnormal 


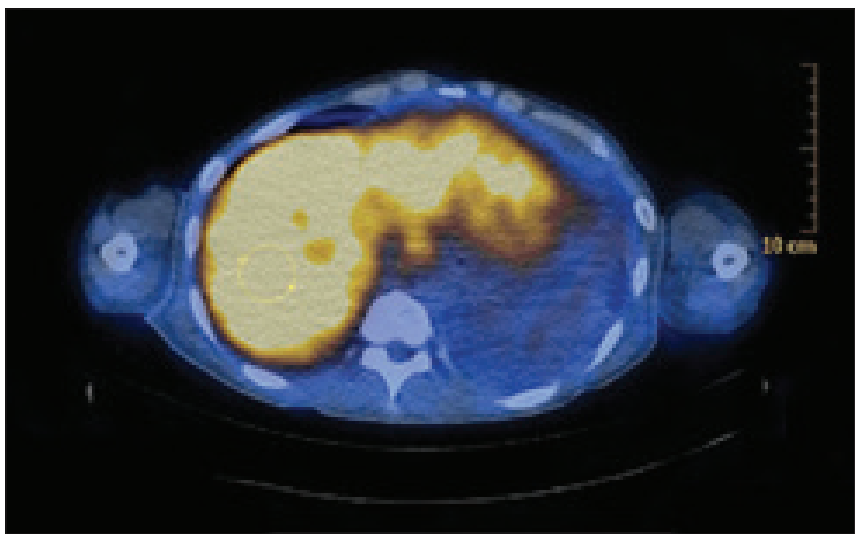

Fig. 3 PET image shows diffuse intense FDG uptake in the liver (SUVmax: 6.6). No increased splenic FDG uptake was seen.

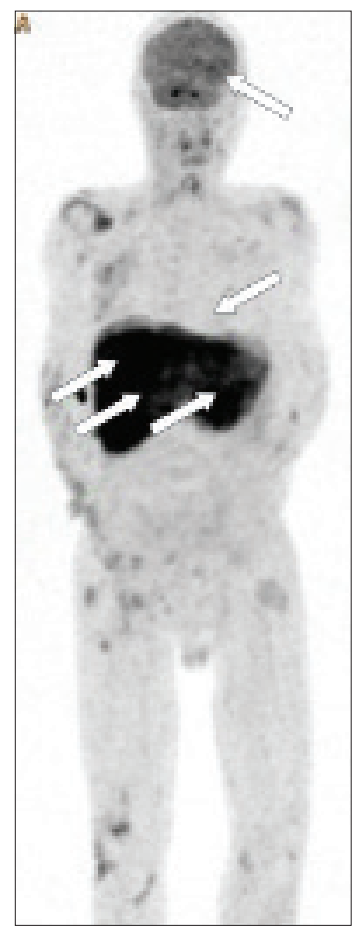

Fig. 4 Whole-body PET image shows diffuse intense FDG uptake in the liver but the physiological intense uptake in the brain and heart is markedly reduced.

FDG uptake was shown in the spleen. However, the normal physiological intense uptake in the brain and heart was markedly reduced (Fig. 4). There were also multiple tiny nodules in both lungs, some of which were arranged in a tree-in-bud pattern. The larger nodules demonstrated mild increase in FDG activity (SUVmax, 2.0) (Fig. 5). Given the diffuse liver parenchymal involvement, lymphoma or tuberculosis was suspected and a liver biopsy was performed. The biopsy showed evidence of granulomatous inflammation, and supplementary Ziehl-Neelsen stain revealed many acid-fast bacilli. A subsequent culture grew Mycobacterium tuberculosis. The patient was consequently started on antituberculous treatment.

\section{DISCUSSION}

Diffuse intense FDG uptake in the liver is an uncommon finding on PET imaging. Most of the cases reported are due
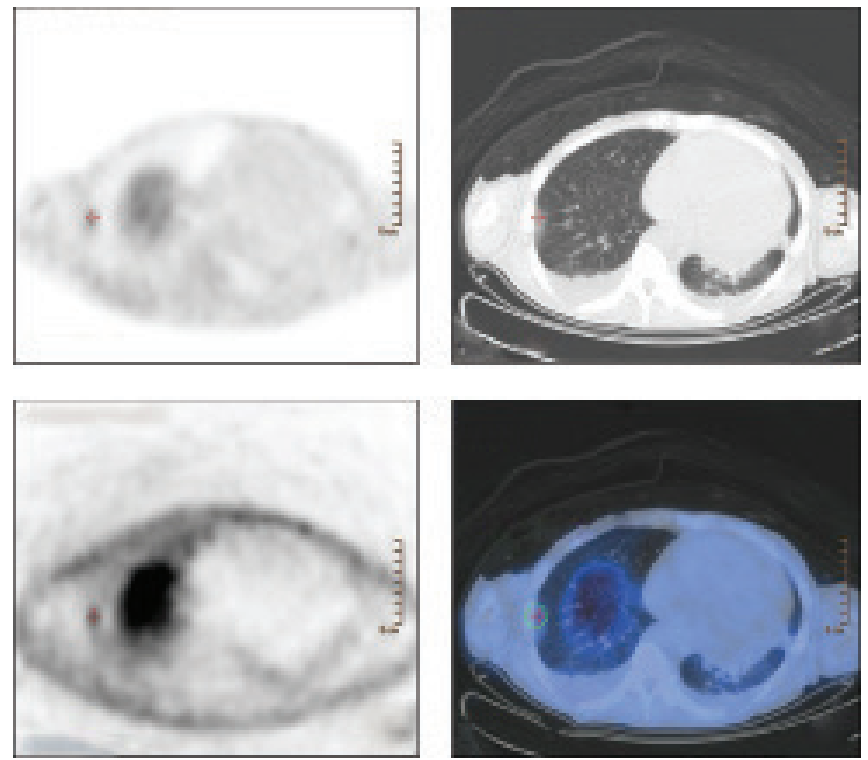

Fig. 5 PET images show multiple tiny lung nodules, with a mild increase in FDG activity demonstrated in a larger nodule in the right lower lobe (SUVmax: 2.0).

to neoplastic conditions, among which the most common is lymphoma. ${ }^{(1-3)}$ Other neoplastic conditions reported to cause FDG hepatic superscan include breast cancer metastases. ${ }^{(4)}$ Infective causes are seldom encountered, with very few case reports describing patients with hepatosplenic tuberculosis ${ }^{(5)}$ and acute mononucleosis ${ }^{(6)}$ presenting with diffuse intense FDG uptake in the liver.

The imaging features of hepatic tuberculosis include hepatosplenomegaly, multiple hepatic nodules, abscess formation or even normal findings. ${ }^{(7,8)}$ In our patient, the biopsy confirmed hepatic tuberculosis, which manifested as diffuse intense FDG uptake in the liver, with markedly reduced physiological uptake in the brain and heart on PET imaging. This phenomenon is similar to the superscan seen in conventional skeletal scintigraphy. FDG hepatic superscan may be attributed to intense glucose uptake by markedly metabolically active disease processes that infiltrate the liver parenchyma. It is a rare manifestation in patients with hepatic tuberculosis, and the possibility of such imaging findings being misdiagnosed as hepatic lymphoma, which commonly presents as diffuse infiltrative liver involvement, cannot be overlooked. ${ }^{(9)}$ A definitive diagnosis of hepatic tuberculosis could be made through liver biopsies, which would reveal granulomatous inflammation and identify acid-fast bacilli, as in the case of our patient.

Multiple lung nodules, some of which were arranged in a tree-in-bud pattern, were seen in our patient, with some of the larger ones being hypermetabolic. These lung nodules probably represent concomitant pulmonary tuberculosis. Unlike a previous report of hepatosplenic tuberculosis, ${ }^{(5)}$ our patient did not have abnormally increased FDG uptake in the spleen, although mild splenomegaly was found on physical examination. This suggests that the spleen was only minimally involved.

It is important that clinicians remain aware of tuberculosis as a differential diagnosis for lymphoma in the face of diffuse FDG 
uptake in the liver on PET imaging, as the clinical management of the two conditions is quite distinct. As overlapping imaging features imply that their differentiation is difficult based on imaging findings alone, liver biopsy should be considered, if clinically warranted, for suitable patients in order to arrive at a definitive diagnosis.

In conclusion, FDG hepatic superscan with reduced physiological activity in the brain and heart, is an uncommon finding on PET imaging. Although it is usually caused by neoplastic lesions (especially lymphoma), hepatic tuberculosis can be a rare cause of FDG hepatic superscan, as in the case of our patient. PET imaging may offer the only viable diagnostic modality for hepatic tuberculosis, as only nonspecific hepatomegaly may be apparent on other imaging modalities. For patients presenting with FDG hepatic superscan, hepatic tuberculosis should be included in the differential diagnosis and liver biopsy should be performed to ensure a definitive diagnosis.

\section{REFERENCES}

1. Itti E, Juweid ME, Haioun C, et al. Improvement of early 18F-FDG PET interpretation in diffuse large B-cell lymphoma: importance of the reference background. J Nucl Med 2010; 51:1857-62.

2. Kaneko K, Nishie A, Arima F, et al. A case of diffuse-type primary hepatic lymphoma mimicking diffuse hepatocellular carcinoma. Ann Nucl Med 2011; 25:303-7.

3. Basu S, Nair N. Unusually elevated liver radioactivity on F-18 FDG PET in Hodgkin's disease: hepatic 'superscan'. Clin Nucl Med 2004; 29:626-8.

4. Tichelaar V, Gemmel F, de Rhoter W, Bronkhorst C, de Graaf H. FDG hepatic superscan caused by massive breast cancer invasion. Clin Nucl Med 2009; 34:716-8.

5. Jeong YJ, Sohn MH, Lim ST, et al. 'Hot liver' on 18F-FDG PET/CT imaging in a patient with hepatosplenic tuberculosis. Eur J Nucl Med Mol Imaging 2010; 37:1618-9.

6. Lustberg MB, Aras O, Meisenberg BR, et al. FDG PET/CT findings in acute adult mononucleosis mimicking malignant lymphoma. Eur J Haematol 2008; 81:154-6.

7. Saluja SS, Ray S, Pal S, et al. Hepatobiliary and pancreatic tuberculosis: a two decade experience. BMC Surg 2007; 7:10

8. Hwang SW, Kim Y], Cho EJ, et al. [Clinical features of hepatic tuberculosis in biopsy-proven cases]. Korean J Hepatol 2009; 15:159-67. Korean.

9. Dähnert W. Radiology Review Manual. 7th ed. Philadelphia: Wolters Kluwer Health/Lippincott Williams Wilkins, 2011. 